\title{
Epistemic transfer between linguistics and neuroscience: Problems and prospects
}

\author{
Giosuè Baggio
}

\begin{abstract}
Language Acquisition and Language Processing Lab, Department of Language and Literature, Norwegian University of Science and Technology, NO-7491 Trondheim, Norway; E-mail: giosue.baggio@ntnu.no
\end{abstract}

\begin{abstract}
This chapter analyzes instances of successful, partial, and failed unidirectional epistemic transfer between theoretical linguistics and neuroscience. I distinguish three types of transfer, depending on the nature of the linguistic knowledge involved: type-A knowledge, about language as such, essentially invariant across theories or formalisms; type-B knowledge, about alternative formal analyses of basic structures and operations in language; type-C knowledge, about the application of various computational methods to analyzing or modeling behavioral or neural data. I conclude that successful epistemic transfer may be achieved, under certain conditions, with type-A and type-C knowledge, and I present some examples of the strengths and limitations of each approach. Type-B transfer, however, in particular from theories of syntactic and semantic composition, so far has not led to new knowledge of the neural correlates and mechanisms of linguistic computation. I suggest that greater theoretical emphasis on algorithmic-level analyses, via a revised notion of linguistic competence and a new model of epistemic transfer, can bring formal linguistics and neuroscience closer together. Finally, I discuss the possible role of a computationalist psycholinguistics as a semi-autonomous 'bridge science', that could serve the aim of linking linguistics and neuroscience through algorithmic models of linguistic functions, aided by emerging methods and results in areas of computational linguistics, computer science, and AI.
\end{abstract}

Prefinal draft. Appears in:

The Philosophy and Science of Language: Interdisciplinary Perspectives. Edited by Ryan M. Nefdt, Carita Klippi. \& Bart Karstens. Palgrave Mcmillan, 2021: 275-308. 


\section{Introduction}

Among the new sciences to have emerged in the early 20th century, linguistics and neuroscience play a special role in the quest for understanding the human mind in scientific terms. The potential for linguistics and neuroscience to jointly illuminate mental structures, both from a formal and a material perspectives, may have been clear before the advent of cognitive science (Levelt 2012). But it is only in the past few decades that fundamental challenges have become visible and that conceptual tools for addressing them have been developed and deployed. Here, I focus on one such challenge, that of effective integration between those two disciplines (Embick \& Poeppel 2015; Poeppel \& Embick 2005), as seen through the conceptual lens of epistemic transfer: the idea that knowledge of language, as produced by linguistics, may be turned over 'as is' to neuroscience and used to generate new knowledge of how language is learned, represented, and processed in the brain. I will argue that, to the extent that integration is a realistic target, unidirectional epistemic transfer (UET) from linguistics to neuroscience may not be the best way to achieve it. I will show that some successful cases of UET actually exist, but other attempts have not led to the desired outcomes. I will introduce two new concepts-deep competence and epistemic pooling-and suggest ways in which they may be used to address the integration challenge. But let us start with a catalogue of transferables, i.e., possible candidates for pure UET drawn from current theoretical approaches to language.

\subsection{Structuralism}

The notions of distinctive feature and of closed systems of linguistic representation based on finite feature sets are lasting contributions of structuralism in linguistics, with important potential consequences for the neurobiology of language. In fact, I will argue below that (apparently) successful instances of UET between linguistics and neuroscience involve precisely distinctive features, in particular in phonology. Traditionally, features are binary, that is, they can take either of two values, + or -, specifying whether a given unit of representation, such as a phonological segment, has (+) or lacks (-) a particular feature (Chomsky \& Halle 1968; Trubetzkoy 1969; Jakobson \& Halle 1971). The actual feature sets being postulated may differ across 
theories, but there remains significant convergence, at least on the ideas of feature subsets describing the voicing, manner, and place of articulation of phonemes, and of phonemes as unique feature combinations: e.g., the consonants /p/ and /b/ can be distinguished by a single feature, VOICE (whether the vocal cords vibrate or not with the articulation of the segment), which is present for $/ \mathrm{b} /$ and absent for $/ \mathrm{p} /$. Closed systems of binary features have been used in parametric analyses of syntax and grammatical acquisition and variation (Chomsky \& Lasnik 1993), and in some decompositional models of semantics, in combination with other representations (Katz \& Fodor 1963; Jackendoff 1983; Pustejovsky 1995). In all these cases, where distinctive features (or equivalent) have been described and applied, it is tempting to assume that linguistics has successfully identified the 'parts list' (Ben Shalom \& Poeppel 2008) of a given system of representation (phonology, syntax, semantics), and that it now falls to neuroscience to identify corresponding 'parts' in the brain: neurons, circuits, areas, or networks that respond preferentially (show sensitivity) to individual distinctive features, or components in neural signals that do so.

\subsection{Formal linguistics}

A second set of transferables can be identified within areas of generative grammar and logical semantics-for lack of a better term, to distinguish them from classical structuralism, I will refer to these fields jointly as 'formal linguistics'. Theoretically, the constructs posited by formal linguistics, that could be candidates for pure UET, are quite different from distinctive features: they are not representational devices, but structure-building operations, such as Merge or Unification in syntax (Chomsky 1995; Shieber 1986/2003) and Functional Application in logical semantics (Heim \& Kratzer 1998; Chierchia \& McConnell-Ginet 2000). These operations are typically at the heart of deductive or derivational formalisms, assumed to model (by design or incidentally) human linguistic competence. In spite of this, these theories often have a characteristic procedural vein, such that core structure-building operations may be accompanied by explicit algorithms for constructing the relevant 'objects', for example syntactic trees, interpretable logical forms, models etc. But even when algorithms or some other computational implementation are not provided, it may 
still be possible to begin a search for the neural correlates of such operations, or at least to evaluate some of the theory's processing consequences. UET here requires linking hypotheses that establish some kind of relationship between complexity of the derivation-within the linguistic theory-and complexity of the process that is (actually or possibly) executed by the brain. Formal linguistics may be assumed to have identified syntactic or semantic composition operations that are necessary to explain human linguistic competence or, to put it in more mundane terms, to make the theory do (tentative) explanatory work. The job of neuroscience then is to find the neural footprints of such operations in language processing experiments.

\subsection{Usage-based approaches}

A third class of approaches in linguistics has recently coalesced into the view that language is not only learned by using it-it is also shaped by usage (Christiansen \& Chater 2016). In these theories, the focus is shifted from the formal structures and operations that constitute competence (e.g., in structuralism and formal linguistics: distinctive features, syntactic and semantic composition etc.) to the processes that construct competence: knowledge of a language is said to be built from experience, which is usually characterized as embodied, enactive, interactive or social etc. This shift should not mislead. Structuralism and formal linguistics may come equipped with theories of learning and performance, as much as usage-based accounts have distinctive views of structure in phonology, syntax, and semantics. Moreover, there are some theories that blend elements of these different traditions, e.g., the theory of language learning by Yang $(2002,2004)$ and the theory of syntax by Culicover $\&$ Jackendoff (2005). However, these approaches diverge sharply on the issue of the balance between storage and computation in the language system. The explanatory strategy used by generative grammar and formal semantics is to assume powerful structure-building operations plus a finite set of building blocks - the 'parts list' of the language: what can be computed is computed, and only what must be stored is stored. In usage-based models, language is seen as a network of constructions, i.e., learned form-meaning pairings, ranging from morphemes to phrasal or sentential constructions: in this account, what can be stored is stored, and only what must be 
computed is computed. More precisely, usage-based accounts place the burden of processing on memory systems in brains and machines, eliminating or minimizing any form of computation other than learning. This view seems to be partly shared by construction grammar ( $\mathrm{CxG}$ ), distributional semantics, connectionist and deep learning network models, and language models as developed in NLP research. The kind of criticism that is often levied, explicitly or implicitly, against neurolinguistic projects built upon generative grammar, logical semantics, and related approaches is that "neuroscience has so far failed to enlighten what we consider to be the core of our linguistic powers, namely the specifically human ability to variably combine meaningful units into rule-conforming higher-order structures" (aka composition; Pulvermüller et al. 2013; Pulvermüller 2010). The relevant candidates for UET are constructions (or, rather, whatever the theory says is stored as a result of learning) and learning mechanisms. The former are directly transferred from linguistics, and the latter are lifted from learning theory, statistics, machine learning etc. The task for neuroscience is to find evidence for constructions in the brain, and to describe language learning in neural systems with specific inductive biases and constraints.

\subsection{Theoretical pluralism}

Considering structuralism, formal linguistics, and usage-based approaches, I have identified three types of candidates for UET between linguistics and neuroscience: distinctive features, structure-building operations, and constructions and learning mechanisms involved in their acquisition. The rest of the chapter is an assessment of recent attempts at searching for the neural correlates or underpinnings of these different linguistic 'objects'. The goal is to evaluate the success of each attempt and to identify specific challenges in cases of partial success or failure. The conclusion, perhaps not surprisingly, will be that UET is hard, even when the transferables can be reliably classified as knowledge, and that, so far, only few attempts at pure UET have been successful. However, even some failed cases of UET can help to generate new knowledge, sparking discovery in the neurobiology of language: of such cases too, I will provide representative examples. 
149 A plea for theoretical pluralism is in order, before we move on. To anyone working 150 in the field of linguistics, or in neighboring fields, it may be clear that language can

151 be rationally reconstructed in multiple ways. Some may be provably equivalent (a 152 respectable industry in linguistics is consecrated to proving equivalence theorems 153 for given grammatical formalisms), others may not, and may therefore be counted 154 as competitors; in that case only, some theories may prevail over others. Even then, 155 different formalisms or theories may gradually converge on similar results, or may 156 blend in ways that reveal complementarity or compatibility of formalisms. A most 157 glaring example in contemporary linguistics is perhaps Jackendoff's evolving view of the 'architecture of language', which incorporates essential elements of all three approaches above. What makes integration of different linguistic ideas possible, in

160 Jackendoff's case, is his general orientation towards cognition and processing. How 161 likely is it that either structuralism, formal linguistics, or usage-based models have 162 hit on 'The One Right Theory of Language in the Brain', given that none of them has been informed by neuroscientific concepts from the outset of theory building, and that none of them makes systematic use of neuroscience results in guiding theory development? What is more likely, at this very early stage of inquiry, is that grains of truth may be found in the best theories from each of the approaches above. The challenge is to find them and tie them together in theoretically coherent ways.

\section{Three scenarios for epistemic transfer}

In what follows, as anticipated above, I will consider a particular form of epistemic transfer between linguistics and neuroscience, discussing alternative models later, in section 3: knowledge transfer that is unidirectional (i.e., between linguistics and neuroscience, but not the other way around-whether or not the latter is possible or currently attested, I will leave open here) and conservative (it leaves knowledge generated in linguistics largely 'as is', i.e., unmodified prior to and during transfer). Additionally, I will accept as largely unproblematic the facts that conservative UET (i) involves true knowledge in the source domain (linguistics), as opposed to either conjectures, beliefs, or constructs with a weaker epistemic status than knowledge; that is not the same as assuming that current theoretical linguistics only generates 
true knowledge, or that the knowledge that is transferred is only knowledge of the object language(s) under study; (ii) that knowledge may effectively be transferred from linguistics to neuroscience in one or several steps, that, however, do not alter the structure or the epistemic status of the source (linguistic) knowledge; (iii) that knowledge transfer fulfils certain epistemic purposes in the target domain, where it may be useful or necessary to build theories and models, to contribute to guiding or constraining experimental design etc., as a result of transfer.

Applying a key distinction from current work in the philosophy of science (Herfeld \& Lisciandra 2019), and elaborating on point (i), knowledge in the source domain can be of three kinds: (A) knowledge about some or all languages, or about human language as a cognitive capacity; (B) knowledge of linguistic formalisms, theories, or models, paired with knowledge that such constructs may be applied to some or all natural languages with some degree of success; (C) knowledge of how to use or apply a set of formal or empirical methods, techniques, or tools from various areas of linguistics (e.g., computational linguistics) to advance research or knowledge in the target domain (neuroscience). Type-A knowledge could provide candidates for realist interpretations of linguistic theories in the target field (e.g., that construct $\mathrm{X}$ is 'neurally real'); type-B knowledge stems from theories that may explain aspects of linguistic competence, but where a realist interpretation in the target domain is limited by the meta-theoretic nature of the knowledge involved; type-C knowledge only provides formal tools that may serve primarily data analysis and occasionally theory or model development in the target domain, but where issues of realism, as is normally understood in the philosophy of science, may not arise. The distinction between these three types of linguistic knowledge that can be transferred to areas of brain science (e.g., cognitive neuroscience, neurology, and neurobiology) is used below to describe three possible scenarios for conservative UET.

\subsection{Scenario 1: Phonological features, syntactic parameters, constructions}

The first scenario considered here includes all cases in which type-A knowledge is available from linguistics, and its transfer to neuroscience leads to discoveries that allow realist interpretations of the relevant linguistic 'objects' and of some of their 
immediate consequences. Distinctive features in phonology are probably the most successful example of type-A knowledge transfer in the neurobiology of language. Mesgarani et al. (2014) studied how speech is represented in areas of the superior temporal gyrus (STG), and specifically whether local neural responses are sensitive to phonemes, to distinctive phonological features, or to low-level spectrotemporal parameters (power in specific frequency bands over time). They recorded cortical surface potentials from STG using implanted electrode arrays in epileptic patients, while they were listening to natural speech samples in English. The authors found that cortical signals from some electrodes were selective for particular phonemes. Yet, the majority of STG electrodes were sensitive not to individual phonemes, but to particular groups of phonemes. This raises the possibility that the neural code in STG represents properties shared by different phonemes, e.g., distinctive features. For instance, among all electrodes that were sensitive to plosives, some responded to place of articulation or voicing features. In general, the data showed grouping of phonemes based on shared features, "suggesting that a substantial portion of the population-based organization can be accounted for by local tuning to features at single electrodes" (Mesgarani et al. 2014; Grodzinsky \& Nelken 2014).

The representation of speech sounds in STG, and generally in the temporal lobe, is complex and still not completely understood, but it most likely involves distinctive features (for recent research and reviews, see Chang et al. 2010; Hickok \& Poeppel 2016; Hullet et al. 2016; Tang et al. 2017; Hamilton et al. 2018; Jasmin et al. 2019). The Mesgarani et al. (2014) data do not show that distinctive features are the only, or the most fundamental, representations of speech, but they do show that features are 'neurally real', since they can be put into strict, systematic correspondence with local cortical responses. If distinctive features (the classical structuralist notion of Jakobson, Trubetzkoy etc.), constitute type-A knowledge-knowledge of language, and not just knowledge of a possible way of coherently reconstructing language-, then these results may count as a successful case of pure UET.

As noted in section 1, closed systems of distinctive features have also been used in parametric syntax and decompositional semantics. However, in those areas, there 
are few studies that systematically assess the 'neural reality' of distinctive features or other units of representation in a linguistic formalism. For instance, research in neuropsychology has (indirectly) addressed some of the basic conceptual features posited by decompositional semantic theories, e.g., the distinctions between living and non-living things, or animate and inanimate entities (Patterson et al. 2007). In those cases, it is impossible to speak of UET between linguistics and neuroscience, because these distinctions are transferred individually, and not as part of a closed system of features. In parametric syntax, one challenge to transfer to neuroscience is that features (parameters: e.g., pro-drop, head directionality etc.) apply to whole languages (and define them), and not to individual linguistic segments, as was the case for distinctive features in phonology. This renders it difficult or impossible to search for the 'parts list' of syntax in the brain (if one accepts a parametric theory) by means of standard language processing experiments. But what is possible is to design stimuli that violate lawful ways of setting some parameters, such as strings where function words (e.g., negation) occupy a fixed linear-order position (e.g., as the $n$-th word). This pseudo-property effectively lies outside the space of principles and parameters that define human languages; such sentences belong instead to an 'impossible language' (Moro 2016). It has been shown that such strings, compared to actual sentences (e.g., in German or Japanese), disengage (i.e., result in reduced activations in) classical language networks, including the left inferior frontal gyrus (LIFG; Musso et al. 2003), and are moreover harder to learn by children (Nowak \& Baggio 2017). Although this is a less clear case than the phonology case, discussed earlier on, it illustrates an alternative approach to type-A epistemic transfer. Here, one is not looking for a correspondence between 'parts lists' - in the theory and in the brain-, but rather for evidence that neural language processing is constrained in ways compatible with the parametric theory (Moro 2019).

The existence of constructions, if not the framework of $\mathrm{CxG}$ as such, is now largely accepted in linguistics. The fact that some sentence types have meanings that may not be derivable compositionally, i.e., are not transparently related to the syntactic structure of the sentence, is an argument for 'listing' those constructions as lexical entries, that is, stored form-meaning pairings (Goldberg 1995, 2003, 2006). These 
should include, at least, idioms and widely attested phrasal constructions, such as sound + motion constructions, beneficiary dative constructions, resultatives, 'X let alone $Y^{\prime}$ constructions, and many more (Culicover \& Jackendoff 2006). Yet, it is not clear whether the radical notion that language is "constructions all the way down" (or up, i.e., that the smallest and the largest structures of language, whatever they are, are the same kind of form-meaning pairings) could serve as a basis for type-A epistemic transfer. Moreover, there is little experimental research in neuroscience on accepted or less controversial grammatical constructions, which could count as type-A knowledge. For instance, Cappelle et al. (2010) report MEG data consistent with the constructionist notion that phrasal verbs (e.g., 'rise up', 'break down' etc.) are not composed syntactically and semantically, but are retrieved as single lexical units. Research using fMRI has shown that idioms engage largely the same regions as literal language (Bohrn et al. 2012; Rapp et al. 2012), but it is not clear whether such activations can be taken as evidence for retrieval of idiom form and meaning, as opposed to also composition, especially if one takes into account factors such as idiom decomposability or semantic transparency (Vulchanova et al. 2019). Finally, the embodied learning and representation mechanisms assumed by most theories in the $\mathrm{CxG}$ camp have been challenged by contrary evidence from studies on single words, phrases, and sentences (Pavan \& Baggio 2013; Papeo et al. 2014; Ghio et al. 2016). In sum, usage-based theories, in particular $C x G$, have generated true type- $A$ knowledge that, importantly, does not also follow from either generative syntax or formal semantics-constructions are real, whether or not one also accepts a broad view of language as "nothing but constructions". However, such knowledge has not yet been successfully transferred to neuroscience. Whether constructions are also 'neurally real', and which phrasal and sentential structures are retrieved as lexical units, are important topics for future work in psycho- and neurolinguistics.

\subsection{Scenario 2: Syntactic and semantic composition}

In the previous section, I have discussed three instances of type-A knowledge, i.e., knowledge about language(s), as such. Distinctive features in phonology, syntactic parameters, and constructions are fairly widely accepted in theoretical linguistics, 
even if one does not endorse in toto the formalisms they are embedded in and the assumptions they make. One hallmark of type-A knowledge is that few theoretical alternatives exist for these particular constructs that are (a) not merely notational variants and (b) empirically equally successful, or more. In this section, I consider type-B knowledge in linguistics: knowledge of linguistic theories (and not directly of language as such) and of their application to modeling specific natural language phenomena. In these cases, there may be well-specified formal constructs, just like type-A knowledge, but conditions (a) and (b) are met. Merge and Unification seem to be good examples, in that these structure-building operations are formally quite different (they are not reciprocal notational variants), yet they seem to have much the same empirical coverage of syntactic structures (they are universal operations, in that sense). Functional Application is another example, the issue being whether or not all semantic composition boils down to it (aka 'Frege's conjecture'; Heim \& Kratzer 1998), or whether additional composition operations must be introduced. Systems with those operations are not merely notational variants of a system with only Functional Application, but the latter may be sufficient to guarantee universal generation of logical forms, given appropriate lexical and syntactic inputs (Heim \& Kratzer 1998; Chierchia \& McConnell-Ginet 2000). Therefore, type-B knowledge is meta-theoretic knowledge of ways of formally reconstructing aspects of syntax and semantics. This invites caution when transferring such knowledge to neuroscience. The question of the 'neural reality' of Merge, Unification, or Functional Application may not be answerable, and should be replaced with the question (given relevant criteria of theoretical coherence, empirical adequacy etc.) of what may be the best account of syntactic and semantic composition in the brain, and what is the role of specific formal operations (Merge, Unification, Functional Application etc.) in such endeavour, in particular at the computational and/or algorithmic levels of analysis (Marr 1982; Baggio et al. 2012a, 2012b, 2015, 2016; Martin \& Baggio 2019).

Questions about the 'neural reality' of structure-building operations in syntax and semantics have been frequently raised in the literature (Townsend \& Bever 2001; Marantz 2005; Embick \& Poeppel 2015; Poeppel \& Embick 2005). Recently, ECoG and fMRI experiments have provided data supporting (or at least consistent with) 
Merge (Nelson et al. 2017; Zaccarella et al. 2017), but none of those studies offers arguments or evidence against Unification (Snijders et al. 2008) or other syntactic structure-building operations with similar coverage and processing predictions. In fact, cortical activations to syntactic structures, against various baselines, typically involving areas of the left inferior frontal gyrus and of the left temporal cortex, can be explained equally well by neurocognitive models assuming Merge (Friederici et al. 2017) and by Unification-based accounts (Hagoort 2003). Merge and Unification may (or may not) be teased apart in other domains than syntax, such as semantics (Hagoort et al. 2009; Baggio \& Hagoort 2011; Baggio 2018). Yet, as models of core syntactic structure-building operations, neither Merge nor Unification have, so far, led to successful UET. We are making progress in identifying the cortical networks underlying syntactic structure building, but this knowledge has not been produced as a result of transferring type-B linguistic knowledge about Merge, Unification etc. to neuroscience. Rather, it results from the implementation of certain experimental designs, which are not strictly based on one specific contemporary theory of syntax, but may be interpreted in the framework of several such theories.

The case of semantic composition is slightly different from the perspective of UET. MEG studies have sought to identify a neural correlate of semantic composition, in the sense of Functional Application, but (so far) without success (Pylkkänen 2019). Pylkkänen and collaborators found stronger MEG responses from the left anterior temporal lobe (LATL) in 'minimal' NPs (e.g., 'red boat') vs word lists containing the same critical noun ('cup, boat'), at about 200-250 msec after noun onset (Bemis \& Pylkkänen 2011, 2013; Blanco-Elorrieta \& Pylkkänen 2016; for EEG data, see Flò et al. 2020, Olstad et al. 2020, Fritz \& Baggio 2020). This early LATL response was initially linked to structure-building operations, but further research by the same lab indicates that it may rather reflect combinatorial processes sensitive to specific conceptual relations between words (e.g., the specificity of the noun relative to the modifying adjective, as in 'spotted fish' vs 'spotted trout'; for a review and critical discussion, see Westerlund \& Pylkkänen 2017, Pylkkänen 2019). So far no evidence has been produced that Functional Application (or some other, formally equivalent composition operation) corresponds to a specific type of neural response: there is 
no evidence, in other words, that Functional Application is 'neurally real'. Still, this does not entail that there is no such thing as a (semantic) composition operation in the brain, or that those attempts at type-B knowledge transfer have been sterile. In fact, even though the 'neural fingerprints' of meaning composition were not found, knowledge was generated about conceptual combination and the role of the ATL in on-line semantic processing, as a result of attempts at UET between linguistics and neuroscience. Perhaps not the success that was hoped for, but not a failure either.

For our purposes, one pressing philosophical question is what distinguishes cases of successful UET for type-A linguistic knowledge (e.g., sensitivity to phonological distinctive features; Mesgarani et al. 2014) from cases in which attempted transfer of type-B knowledge leads to novel empirical results, but not necessarily to tighter integration of linguistics and brain science (e.g., studies showing that syntactic or conceptual-semantic processing have specific neural correlates, without, however, providing sharp evidence for the 'neural reality' of one particular formal operation, as envisaged by linguistics, such as Merge, Unification, Functional Application etc.). I cannot attempt a definite answer here. This is a deep problem our field is likely to struggle with for some years to come. But it seems the distinction between type-A and type-B knowledge should be part of the answer. There is a difference between knowing that phonemes, across languages, are defined by distinctive features, that the grammar of languages varies parametrically within limits, and that some types of construction exists (type-A), vs knowing that syntactic or semantic composition can be formalized as Merge or Unification or Functional Application (type-B). This is precisely the difference between knowledge of language and knowledge of ways of (formally) reconstructing language. Attempts at either type-A or type-B transfer can lead to generation of new knowledge in neuroscience, but only the former can, and perhaps only in exceptional circumstances, establish the 'neural reality' of the relevant linguistic 'objects'. Type-B epistemic transfer can, at most, provide formal frameworks within which experimental data may be interpreted, without however adjudicating between basic constructs (e.g., Merge vs Unification). What is at stake here, given the distinction between type- $A$ and type-B knowledge, is precisely the issue of scientific realism for neurolinguistics and the neurobiology of language: i.e., 
in what conditions may one take a realist stance on particular linguistic constructs in the context of theories of language in the brain.

\subsection{Scenario 3: Computational linguistics and neural network models}

The third scenario for UET between linguistics and neuroscience relates to the use of methods, techniques, and tools from computational linguistics and neighboring fields, primarily as aids in the analysis of experimental behavioral and neural data, and secondarily as support in theory and model development in the neurobiology of language. The type-C knowledge involved in these cases is knowing how certain methods, techniques, or tools work, when applied to relevant data types, the kinds of inferences they support, and their limitations (given performance benchmarks). Often, type-C knowledge includes type-A and/or type-B knowledge from linguistic theory (e.g., NLP models of parsing and interpretation necessarily make abundant use of linguistic notions). But what gets transferred in this case is knowledge of the method as such. That is what is used to generate new knowledge in the target area, usually to discover patterns in neural data. Recently, some authors have argued for the need for tighter integration or interplay between the neuroscience of language and computational linguistics and NLP/AI research (Hasson et al. 2018; Hasson \& Nusbaum 2019). Recent studies have used methods from computational research on parsing (constructing syntactic representations of sentences) in the analysis of MEG data from language processing experiments (Brennan \& Pylkkänen 2017), or in classifying known ERP responses to sentences (Michalon \& Baggio 2019). Other work has used language models and distributional semantic models to investigate the effects of word predictability and semantic similarity on neural signals (Frank \& Willems 2017). All these studies, via modeling, have revealed patterns in M/EEG or fMRI data that standard statistical analyses would be blind to: for example, that responses from the LATL reflect a predictive left-corner parsing strategy (Brennan \& Pylkkänen 2017); that P600 effects in ERP data can index a conflict between the assignment of grammatical roles (e.g., subject and object) by autonomous, parallel meaning and grammar systems (Kim \& Osterhout 2005; Michalon \& Baggio 2019); that word predictability and semantic similarity are correlated with activations in 
different brain networks, though they leave similar traces on ERP signals (Frank \& Willems 2017). In these cases, type-C epistemic transfer is largely successful: new knowledge about language processing in the brain is generated, strictly as a result of the application of computational linguistics methods to neural data. However, it is clear that the methods used are 'just' tools for discovering or modeling patterns in data, and are not representations (theories or models) of the relevant biological systems at work: the brain is not (or does not contain) a classifier, or a probabilistic language model, or a dependency parser. In type-C epistemic transfer, questions of realism do not really arise. Rather, instrumentalism is the default interpretation of the 'objects' (methods) transferred from linguistics to brain science.

It is important to emphasize the limitations of this approach, particularly vis-à-vis the detection of patterns in data that do not correspond to (and are not correlated with) any construct or component in the models being applied. Although methods from computational linguistics, NLP, machine learning, and AI are often extolled as excellent discovery tools, greatly extending the human capacity to find patterns in data, they are only as powerful as the formal linguistic assumptions they embody, from a representational and computational perspectives. Recent work by Lyu et al. (2019) illustrates this point well. Their goal was to study "the neural mechanisms underpinning semantic composition" (in the sense above), through a combination of experimental M/EEG data and computational linguistics tools. Composition, qua Functional Application, is guided by syntax; therefore, functions and arguments are independent - there are no relations or constraints between them that can facilitate or hinder, allow or block composition. ${ }^{1}$ As I noted above, research so far has failed to find a neural correlate of semantic composition. Lyu et al. used topic modeling, an NLP technique, to capture statistical relationships between subjects, verbs, and objects in sentences, based on corpus data: some verbs constrain their object noun more than others, e.g., 'eat' selects something edible as a direct object noun, while

\footnotetext{
${ }^{1}$ This holds only for arguments of the appropriate logical type. Obviously, constraints exist on which functions may be applied to which logical argument types. For examples, see the extensive literature on type mismatches, type shifting, and coercion (Pustejovsky 1995; Heim \& Kratzer 1998).
} 
'want' places fewer constraints on object nouns. The main result is that activation in left perisylvian cortex is best explained by a model that takes these constraints into account, and not by a model that only captures the contribution of the object noun, independent of the context in which it occurs. The time course of the effect, the networks engaged, and the context-sensitive nature of the process all support current models of semantic activation in the brain (see Hagoort et al. 2009; Baggio \& Hagoort 2011; Baggio 2018). This is an important finding, but it does not reveal either the "neural mechanisms" or the "neural dynamics of semantic composition", because composition is not context-sensitive in the way most topic models are, and requires instead independent representations of functions and arguments (Martin \& Doumas 2019). Computing conditional probabilities in topic models (in general, in language models) is quite different from composition in formal or distributional semantics, which involves logical or algebraic operations on constituent meanings. If compositional dynamics were present in the data, topic models would simply be blind to them, precisely because these models do not embody the representations and operations formally involved in syntactic or semantic composition.

Another example of the limitations of current computational models as applied to neural data is provided by recent research by Rabovsky et al. (2018), describing a connectionist model of the N400 component and effect in ERPs. More specifically, they model semantic processing as the probabilistic updating of sentence meaning representations in a connectionist network. Their simulations reproduce as many as 16 known instances of the N400 effect qualitatively, and other data points (e.g., discourse-level N400 effects) seem fully within the model's reach. This impressive performance sets a new benchmark for future algorithmic-level models of semantic processing and for neural-level models of the N400. Their long-term success must, however, be measured by their capacity to reproduce all and only the N400 effects reported in the literature. It is now largely accepted that the N400 reflects aspects of lexical semantic access and integration, and recent studies show that the N400 has complex functional and spatio-temporal characteristics (see Baggio \& Hagoort 2011 for a theory and Nieuwland et al. 2019 for supporting data from a large-scale study). However, the challenge posed by N400 results that were originally taken to 
back up the integration account remains. It could be instructive to train and test the model on stimuli from studies on quantifiers, event structure, negation, and other 'hard cases' for simple activation theories of the N400 (Urbach et al. 2015; Ferretti et al. 2007; Baggio et al. 2010; Kuperberg et al. 2010). These would be important steps toward assessing the model's empirical adequacy more thoroughly. Perhaps the most probative question is not whether Rabovsky's model is powerful enough, but whether it is 'too powerful'. The representations that are dynamically encoded and updated in the network are "implicit", in that they lack any predefined formal structure, unlike traditional models in linguistic semantics. Moreover, there are no bounds on the kinds of semantic contents that the network may come to represent. Such constraints, the authors argue, may well be "an impediment to capturing the nuanced, quasiregular nature of language" (Rabovsky et al. 2018). Yet, their model may overgenerate for precisely this reason: it may predict N400 effects where none are found experimentally. Studies have reported sustained negative ERPs different from the N400-in latency, duration, and scalp distribution-in reaction to critical words in sentences that trigger 'updates' of event representations in imperfective, aspectual coercion, and light verb constructions (see Baggio et al. 2008; Paczynski et al. 2014; Wittenberg et al. 2014). Their model should also be trained and tested on stimuli from these studies. If semantic updates in their model are specific to the processes underlying the $\mathrm{N} 400$, there should be no differences in activation levels, in the model, before and after presentation of the words that elicit sustained ERPs. These considerations show that applications of type-C knowledge to experimental data should still meet basic requirements of empirical adequacy to lead to effective knowledge transfer and generation of new knowledge in neuroscience: the chosen probabilistic update algorithm can only be considered a theory of the mechanisms behind the N400, if the model captures all and only known instances of the effect.

\section{Deep competence and epistemic pooling}

In section 2, I have argued that type-A and type-C linguistic knowledge may lead to production of new knowledge in the neuroscience of language, as the direct result of transfer. Typically, this new knowledge concerns the 'neural reality' of linguistic 
constructs (type-A) and patterns in behavioral or brain data (type-C). The problem for type-A transfer is to identify 'true' linguistic knowledge among the conjectures, hypotheses, or other constructs generated by theoretical linguistics: that would be knowledge (i) invariant across formalisms (equal alternatives are mere notational variants), (ii) widely accepted in the linguistic community, and (iii) such that there are no competitors with equal or greater explanatory scope and power-these are all hallmarks of a 'mature' theory, according to modern philosophy of science. The challenge for type-C transfer is to select appropriate data analytic methods among those currently available, and to resist realist interpretations of the constructs that are being applied in data analysis or modeling.

As for type-B transfer, different and seemingly harder problems arise, particularly if we consider knowledge from generative grammar, logical semantics, and related theoretical frameworks. Currently, much research in the neuroscience of language operates with formal linguistics as/in its background, but whatever results may be generated, they are not strictly and directly the outcome of knowledge transferred from that background. As an example, several fMRI studies have converged on the description of a left-hemispheric perisylvian network for basic structure building, regardless of whether these studies assumed Merge or Unification (quite different operations formally) as part of their background, i.e., as if one's preferred syntactic theory did not matter for the purposes of designing and conducting neuroimaging experiments that may reliably identify language- or syntax-relevant brain regions. There seem to be, in other words, factors (conceptual or empirical) preventing our best theories in formal linguistics to make direct contact with experimental neural data. Why have we not found any robust brain correlates, let alone mechanisms, of syntactic and semantic composition as defined by formal linguistics? Why, when we look for those correlates, do we invariably find brain responses with very different characteristics from those formal linguistics has ascribed to syntactic and semantic composition-i.e., graded, probabilistic, context-dependent, even predictive, when the latter are discrete, algebraic, context-independent, and bottom-up? 
540 At present, this is one of the most obvious puzzles in the neurobiology of language, 541 and it concerns primarily type-B knowledge from formal linguistics. Some authors 542 have argued that, effectively, generative syntax has already been integrated within 543 the neuroscience of language (e.g., Marantz 2005, Friederici 2017), but many seem 544 to share a more pessimistic outlook. Here, I suggest two strategies for dealing with 545 this predicament, based on the concepts of deep competence and epistemic pooling, 546 both meant to support type-B transfer. None of them is a proven strategy, but both 547 are fairly straightforward next steps in the search for alternatives to type-B UET.

\subsection{Deep competence}

549 One epistemological obstacle to further integration between theoretical linguistics 550 and neuroscience is a particular notion of linguistic competence as (a) knowledge of a formal deductive or derivational system for the generation and interpretation of linguistic structures, (b) distinct from processing and 'performance' (Jackendoff 2002; Baggio et al. 2012a). The problem is whether and how a derivational system (e.g., a formal theory of logico-semantic composition based on the $\lambda$-calculus) could be transferred to a target domain where the goal is to develop processing theories, as in psycholinguistics, neuroscience, computer science or AI etc. Transferring that knowledge 'as is' (conservative transfer) and qua deductive system would miss the point, especially if the goal is to apply it to study physical machines, such as brains, where computation is not (all) deductive. The strategy used by 'strict competence' (Steedman 1992), according to which processing algorithms are as determined by the competence theory and, therefore, computation is deductive, is not viable: the well-documented early failure of the so-called derivational theory of complexity in syntax (DTC) testifies to that (Fodor \& Garrett 1966, 1967). Here is the fork in the road: either we drop the requirement or desideratum that competence theories or models are or involve deductive formalisms, effectively adapting them to demands from the target domain (constraint-based formalisms in linguistics may be seen as instances of this approach, e.g., Jackendoff 2007), or we adopt a 'weak competence' stance, such that the structures being computed (syntactic trees or graphs, logical forms, models etc.) are those sanctioned by one's (deductive) competence theory, 
but computation is not deductive. Either way, we are compelled to take processing seriously, and examine how a theory of competence-whether deductive or based on some other formalism-may be implemented algorithmically and neurally.

Deep competence is precisely the idea that what matters for the study of linguistic (syntactic and semantic) competence is not what formalisms one adopts at the top level of analysis (Marr \& Poggio 1976; Marr 1982), but what aspects and elements of one's top-level (linguistic) theory are implemented algorithmically and neurally. So, linguistic competence is not just declarative knowledge (e.g., knowledge of the syntactic and semantic 'facts' of a language), but also procedural knowledge (e.g., of how to compute form and meaning, resolve ambiguities etc.; Baggio 2020), so long as it can be plausibly and tractably implemented in models of brain processes. One important advantage of this perspective is that now linguistic theories need not be accepted or rejected wholesale as competence theories. We can distinguish, within the top-level computational theory (i.e., the formal linguistic theory as such), which aspects are implementable, and are therefore part of deep competence, and which elements support the competence theory 'from the outside', providing constraints on the computation: these may affect what the system does (or what it can do), but are not part of what it 'knows' (i.e., of deep competence). Similarly, Poggio (1981) and Marr (1982) have argued that a computational-level theory of vision requires a precise characterization of the "properties of the visible world that constrain the computational problem". Key aspects of the structure of the physical world should be "incorporated as critical assumptions of the computation", which is required to make computational problems "well defined and solvable". The same would apply to theories of syntax and semantics. The role that some formal notions play in the theory would be restricted to the computational level, without however being part of what the system effectively computes. Notions of truth and entailment, e.g., truth conditions tout court, may be an example. Those constructs are necessary to make (some versions of) semantic theory work, also qua theory of competence, but they cannot be reasonably assumed to be implemented in the brain, if only because the logical formalisms in which such notions are typically specified lack the properties 
600 (e.g., decidability) that would make them algorithmically implementable (Frixione 601 2001; Partee 2018; Baggio 2020).

602 How could these ideas facilitate or help clarify the nature of the problems involved 603 in type-B epistemic transfer? Deep competence may be seen as a filter on transfer: 604 only those formal constructs that are implementable, algorithmically and neurally, 605 will be transferred to the target domain-we may assume that the goal of transfer to neuroscience is to establish 'neural correlates' of (or mechanisms associated to) some of those constructs, e.g., Merge, Move, Unification, Functional Application etc. The intuitive notion of 'implementability' should be broken down into appropriate technical concepts from computational complexity theory, the most consequential of which may be tractability. Syntactic and semantic composition, as implemented in the brain, are constrained by its finiteness - the fact that there are limited space (memory) and time resources for computation. Therefore, the functions describing composition should be computationally tractable, i.e., a subset of polynomial-time computable functions, plus some super-polynomial-time computable functions for which one or more input parameters are small in practice (van Rooij 2008). There is little formal work applying these specific computational constraints to modeling language processing, but the importance of this approach, as a preliminary step in epistemic transfer, should be clear: knowledge about composition in the brain (let us assume there is such a thing) can be generated as a direct result of transfer, only if tractable composition operators are considered. ${ }^{2}$ Note, however, that tractability does not guarantee that the resulting implementation is the true one. For instance, it may be that making the relevant composition functions tractable has unpleasant consequences for the system's implementational architecture (e.g., for the number, nature, and interrelation of the proposed algorithms; for discussions about syntax, and Merge in particular, see Frampton \& Gutman 1999, Petersson \& Hagoort 2012,

\footnotetext{
${ }^{2}$ Among the computational problems whose combinatorial search spaces may be super-polynomial, van Rooij (2008) mentions one that is different from composition, but sufficiently closely related to deserve attention: i.e., the Input is a surface form $s$, a lexicon $D$, and a lexical-surface form mapping mechanism $M$, and the Output is a set of lexical forms $U$ generated by $D$ from which $M$ can create $s$ (Barton et al. 1987; Ristad 1993).
} 
Trotzke \& Zwart 2014). So, tractability is surely necessary, but not sufficient: other factors may constrain algorithmic and neural implementation, such as plausibility of the resulting architecture, learnability and evolvability of the relevant functions etc. (van Rooij \& Baggio 2020). These computational considerations may guide UET between formal linguistics and neuroscience, in particular when type-B knowledge, or 'theoretically loaded' linguistic knowledge more generally, is concerned.

\subsection{Epistemic pooling}

Deep competence suggests a fairly straightforward strategy for narrowing the gap between linguistic constructs and neural reality: only constructs that are provably implementable (e.g., tractable functions or operators) may count as candidates for type-B UET. This move is designed to reduce the initial set of transferables a priori and strictly on computational grounds, so that empirical or experimental research can proceed with testing fewer but formally better specified ideas.

The second strategy I sketch out here is compatible with the first one, in that it too emphasizes the need to work at the algorithmic-and-architectural level of analysis to achieve better integration between linguistics and neuroscience. However, this second proposal modifies the very idea of epistemic transfer. As before, I consider epistemic transfer, both UET and its new modified version, in the context of Marr's (1982) multi-level framework for the analysis of information processing systems: epistemic transfer corresponds to devising algorithmic- and neural-level analyses that effectively implement a given computational (i.e., linguistic) theory. According to the traditional 'functionalist' approach, one starts at the top of the scheme, from the computational level, and works one's way 'down' to the algorithmic and neural levels-in that order. Because stating the computational problem is a prerequisite for algorithmic analyses, this is obviously the correct approach. But it is much less clear that the same kind of relationship of logical priority should hold between the algorithmic and neural levels of analysis. If we accept this notion (and here I argue that we needn't and shouldn't), we run against two problems. The first is the curse of multiple realization, meaning that, much as the same function may be computed by different algorithms, the same algorithm may be executed by different physical 
machines, possibly with different architectures, components etc. This problem can only be addressed, or at least mitigated, by taking into account neural constraints, for example, from experimental research or, in any case, from the 'outside' of one's processing theory. A second issue is that, ironically, formalism can be an 'iron cage'. The workings of even the smallest physical components of a system, at the lowest of its levels of organization, may be described algorithmically: single 'neurons' are a classic case (McCulloch \& Pitts 1943), but the same applies, for example, even to ion channels (e.g., see van Dongen 1996). This blurs the line between algorithmicand neural-level descriptions, which may well be a virtue-the blurrier the line, the fewer the gaps to be filled-, but still poses the problem of integrating information from experimental measurements, which are typically continuous, dynamic, noisy etc., different from algorithmic model outputs. These issues point to the challenges of anchoring one's algorithmic theory in neurobiology strictly from the top down.

Epistemic pooling is a special case of transfer, which is not unidirectional and does not just involve one source (linguistics) and one target (neuroscience) domains. In epistemic pooling, knowledge is transferred from two domains into a third one: in this case, from linguistics and neuroscience into a field of inquiry equidistant from both (i.e., not a branch of linguistics or a branch of neuroscience), autonomous but capable of assimilating linguistic and neuroscientific knowledge, and emphasizing theory at Marr's intermediate level of analysis, that is, the level of representations, algorithms, and architectures. A computationalist psycho-/neurolinguistics may fit this rough characterization reasonably well (for an early programmatic statement, see Arbib \& Caplan 1979). Developing verisimilar algorithmic-level analyses of the problems or tasks involved in language processing requires pooling of knowledge from linguistic theory (specifying what functions are computed) and neuroscience (constraining the implementation of the relevant computations; for example, how much representational redundancy and architectural parallelism may be involved, see Michalon \& Baggio 2019 for a case study). This strategy is effectively a middle ground between a functionalist approach to modeling (it saves the mathematically necessary computational $\rightarrow$ algorithmic link) and the newer neuroscience-oriented bottom-up stances (it reverses the algorithmic $\rightarrow$ neural link, allowing neural-level 
constraints to co-determine algorithmic-level analyses; Hasson et al. 2018). There currently is a paucity of research using this specific approach, particularly on topics involving type-B linguistic knowledge about composition(ality) and related issues. However, greater theoretical emphasis and analytic efforts placed on intermediate levels of analysis are likely to stimulate pooling of knowledge from both linguistics and neuroscience, and perhaps eventually greater integration between the two. ${ }^{3}$

\section{Conclusion}

Language has a puzzling 'dual nature': as the object of theoretical linguistics, it is a system defined by characteristic formal structures and operations; as the object of human neuroscience, it is a complex, integrative function implemented via specific neurophysiological mechanisms in the brain. Convergence between linguistics and neuroscience is often viewed as a desirable goal, but so far achieving it has proved exceedingly hard. Because the concepts, methods, and background assumptions of linguistics and neuroscience are so different, their results, although fundamentally about the same object of inquiry-i.e., language as a human mental capacity-, are not immediately integratable. Moreover, and for similar reasons, neither discipline seems able to adapt to the other to an extent sufficient to enable integration. Here, I have argued that this onerous task falls to disciplines operating in areas between linguistics and neuroscience-primarily psycholinguistics, but also newer research endeavors (e.g., in computational linguistics, AI etc.) whose theoretical constructs fit the bill of Marr's algorithmic theory, that is, interfacing computational-level and physical-level descriptions of the given cognitive systems at work. This perspective confers on psycholinguistics the status of a 'bridge science'. Although it used to be

\footnotetext{
${ }^{3}$ A precursor of this approach is the co-evolutionary stance adopted by Churchland, Sejnowski, and others. I share with that proposal the notions that (1) algorithmic analyses are formally independent of implementational theories (e.g., because of multiple realizability), but discovery of the algorithms underlying cognition or behavior will depend on understanding aspects of the structure and function of relevant brain systems (Churchland \& Sejnowski 1988, 1992), and (2) one goal of neuroscience is the discovery of such algorithms, as opposed to the study of how independently-assumed algorithms could be physically or biologically realized (Sejnowski et al. 1988).
} 
a 'frontier science', driving research forward in early modern linguistics and brain sciences (aphasiology and neurology) (Levelt 2012), it may now be best construed as a semi-autonomous field, which can achieve the goal of re-connecting linguistics and neuroscience only by building on high-quality results from both disciplines, as is implied by the concept of epistemic pooling introduced here. ${ }^{4}$

I have argued that integration between linguistics and neuroscience may occur via unidirectional, conservative epistemic transfer, but only if the linguistic knowledge involved is either directly about language (type-A) or about methods applicable to behavioral or neural data analysis and data modeling (type-C). Knowledge of ways of formalizing linguistic structures and operations (type-B) is unlikely to generate new knowledge about how language is represented and processed in the brain, as is testified by many recent problematic attempts at identifying neural correlates or mechanisms of syntactic and semantic composition operations, as defined by formal linguistic theories. Greater emphasis on tractable, plausible composition functions, and on neural constraints on their algorithmic implementation, may help build the missing bridges between type-B linguistic knowledge and human neurobiology. As I have suggested here, this move requires that we revise our concepts of linguistic competence (deep competence) and epistemic transfer (epistemic pooling), so as to re-focus theoretical efforts on algorithmic-level analyses. This has two effects. The first is that 'mature' theories of language in the brain will be algorithmic. They will be neither versions of current linguistic theories, whose core formal constructs are replaced with 'neurally plausible' ones (e.g., vectors instead of symbols), nor giant computer simulations of the brain 'doing language'. The second is that none of the currently dominant epistemological stances results in a viable methodology for the present era of language science: functionalism and rationalism (formal linguistics)

\footnotetext{
${ }^{4}$ This account of psycholinguistics is an application of a broader view of human cognitive psychology as a semi-autonomous field, whose primary epistemic purpose is to integrate results from the formal and biological sciences, relevant to understanding human behavior. Philosophically, this also entails that one may deconstruct and reconstruct the (metaphysical) category of mind in precisely the ways that serve best the bridging between the formal sciences (logic, linguistics, probability, decision and game theory etc.) and the biological sciences (neuropsychology, neurobiology, neurogenetics etc.).
} 
will at best give us computational-algorithmic models untethered to neural reality, while physicalism and empiricism (neuroscience, usage-based linguistics) might be ultimately unable to extract from learning models a complete account of linguistic capacities. What may be more likely to succeed, given the arguments articulated in this essay, is an approach that weds minimal but strong structural assumptions on the search for the algorithms that compute the linguistic functions of interest (e.g., tractability analyses) with neurobiological constraints on implementation, taking seriously the idea that characteristics of the brain co-determine, together with the computational problem as such, the algorithms, representations, architectures, and mechanisms that constitute the language system functionally.

Acknowledgements. I am grateful to Yosef Grodzinsky, Richard Lewis, Andrea E. Martin, James McClelland, Andrea Moro, Iris van Rooij, and Mathew Walenski for inspiration, input, and feedback on the ideas presented here.

\section{References}

Arbib, M. A., \& Caplan, D. (1979). Neurolinguistics must be computational. Behavioral and Brain Sciences, 2(3), 449-460.

Baggio, G. (2018). Meaning in the Brain. Cambridge, MA: MIT Press.

Baggio, G. (2020). Deep competence and semantic theory. Mind \& Language.

Baggio, G., Van Lambalgen, M., \& Hagoort, P. (2008). Computing and recomputing discourse models: An ERP study. Journal of Memory and Language, 59(1), 36-53.

Baggio, G., Choma, T., van Lambalgen, M., \& Hagoort, P. (2010). Coercion and compositionality. Journal of Cognitive Neuroscience, 22(9), 2131-2140.

Baggio, G., \& Hagoort, P. (2011). The balance between memory and unification in semantics: A dynamic account of the N400. Language and Cognitive Processes, 26(9), 13381367.

Baggio, G., van Lambalgen, M., \& Hagoort, P. (2012a). Language, linguistics and cognition. In Kempson, R., Fernando, T., \& Asher, N. (Eds.), Philosophy of Linguistics. Amsterdam: Elsevier, 325-355.

Baggio, G., van Lambalgen, M., \& Hagoort, P. (2012b). The processing consequences of compositionality. In Hinzen, W., Machery, E., \& Werning, M. (Eds.), The Oxford Handbook of Compositionality. Oxford: Oxford University Press, 655-672. 
Baggio, G., van Lambalgen, M., \& Hagoort, P. (2015). Logic as Marr's computational level: Four case studies. Topics in Cognitive Science, 7(2), 287-298.

Baggio, G., Stenning, K., \& van Lambalgen, M. (2016). Semantics and cognition. In Aloni, M., \& Dekker, P. (Eds.), The Cambridge Handbook of Formal Semantics. Cambridge: Cambridge University Press, 756-774.

Barton, G. E., Berwick, R. C., \& Ristad, E. S. (1987). Computational Complexity and Natural Language. Cambridge, MA: MIT Press.

Bemis, D. K., \& Pylkkänen, L. (2011). Simple composition: A magnetoencephalography investigation into the comprehension of minimal linguistic phrases. Journal of Neuroscience, 31(8), 2801.

Bemis, D. K., \& Pylkkänen, L. (2013). Basic linguistic composition recruits the left anterior temporal lobe and left angular gyrus during both listening and reading. Cerebral Cortex, 23(8), 1859-1873.

Blanco-Elorrieta, E., \& Pylkkänen, L. (2016). Composition of complex numbers: Delineating the computational role of the left anterior temporal lobe. NeuroImage, 124, 194-203.

Ben Shalom, D., \& Poeppel, D. (2008). Functional anatomic models of language: Assembling the pieces. The Neuroscientist, 14(1), 119-127.

Bohrn, I. C., Altmann, U., \& Jacobs, A. M. (2012). Looking at the brains behind figurative language-A quantitative meta-analysis of neuroimaging studies on metaphor, idiom, and irony processing. Neuropsychologia, 50(11), 2669-2683.

Brennan, J. R., \& Pylkkänen, L. (2017). MEG evidence for incremental sentence composition in the anterior temporal lobe. Cognitive Science, 41, 1515-1531.

Cappelle, B., Shtyrov, Y., \& Pulvermüller, F. (2010). Heating up or cooling up the brain? MEG evidence that phrasal verbs are lexical units. Brain and Language, 115(3), 189-201.

Chang, E. F., Rieger, J. W., Johnson, K., Berger, M. S., Barbaro, N. M., \& Knight, R. T. (2010). Categorical speech representation in human superior temporal gyrus. Nature Neuroscience, 13(11), 1428.

Chierchia, G., \& McConnell-Ginet, S. (2000). Meaning and Grammar: An Introduction to Semantics. Cambridge, MA: MIT Press.

Chomsky, N., \& Halle, M. (1968). The Sound Pattern of English. New York: Harper \& Row.

Chomsky, N., \& Lasnik, H. (1993). The theory of principles and parameters. In: J. Jacobs, et al. (Eds.), Syntax: An International Handbook of Contemporary Research, 1, Walter de Gruyter (1993), pp. 506-569.

Christiansen, M. H., \& Chater, N. (2016). Creating Language: Integrating Evolution, Acquisition, and Processing. Cambridge, MA: MIT Press. 
801 Churchland, P. S., \& Sejnowski, T. J. (1988). Perspectives on cognitive neuroscience. Science, 802 242(4879), 741-745.

803 Churchland, P. S., \& Sejnowski, T. J. (1992). The Computational Brain. Cambridge, MA: MIT 804 Press.

805 Culicover, P. W., \& Jackendoff, R. S.,(2005). Simpler Syntax. Oxford: Oxford University Press.

806 Embick, D., \& Poeppel, D. (2015). Towards a computational(ist) neurobiology of language: 807 Correlational, integrated and explanatory neurolinguistics. Language, Cognition and 808 Neuroscience, 30(4), 357-366.

809 Ferretti, T. R., Kutas, M., \& McRae, K. (2007). Verb aspect and the activation of event 810 knowledge. Journal of Experimental Psychology: Learning, Memory, and Cognition, 33(1), 811182

812 Fló, E., Cabana, Á., and Valle-Lisboa, J. C. (2020). EEG signatures of elementary composition: 813 Disentangling genuine composition and expectancy processes. Brain and Language, 209, 814104837.

815 Fodor, J., \& Garrett, M. (1966). Some reflections on competence and performance. In Lyons, 816 J., \& Wales, R. J. (Eds.), Psycholinguistics Papers. Edinburgh: University of Edinburgh Press, $817 \quad 135-154$.

818 Fodor, J., \& Garrett, M. (1967). Some syntactic determinants of sentential complexity. 819 Perception and Psychophysics 2: 289-296.

820 Frampton, J., \& Gutmann, S. (1999). Cyclic computation, a computationally efficient 821 minimalist syntax. Syntax, 2(1), 1-27.

822 Frank, S. L., \& Willems, R. M. (2017). Word predictability and semantic similarity show 823 distinct patterns of brain activity during language comprehension. Language, Cognition 824 and Neuroscience, 32(9), 1192-1203.

825 Friederici, A. D., Chomsky, N., Berwick, R. C., Moro, A., \& Bolhuis, J. J. (2017). Language, mind 826 and brain. Nature Human Behaviour, 1(10), 713-722.

827 Fritz, I., \& Baggio, G. (2020). Meaning composition in minimal phrasal contexts: Distinct 828 ERP effects of intensionality and denotation. Language, Cognition and Neuroscience, 35(10), $829 \quad 1295-1313$.

830 Frixione, M. (2001). Tractable competence. Minds and Machines, 11(3), 379-397.

831 Ghio, M., Vaghi, M. M. S., Perani, D., \& Tettamanti, M. (2016). Decoding the neural 832 representation of fine-grained conceptual categories. Neuroimage, 132, 93-103.

833 Goldberg, A. E. (1995). Constructions: A Construction Grammar Approach to Argument 834 Structure. Chicago: University of Chicago Press. 
Goldberg, A. E. (2003). Constructions: A new theoretical approach to language. Trends in Cognitive Sciences, 7(5), 219-224.

Goldberg, A. E. (2006). Constructions at Work: The Nature of Generalization in Language. Oxford: Oxford University Press.

Grodzinsky, Y., \& Nelken, I. (2014). The neural code that makes us human. Science, 343(6174), 978-979.

Hagoort, P. (2003). How the brain solves the binding problem for language: a neurocomputational model of syntactic processing. Neuroimage, 20, S18-S29.

Hagoort, P., Baggio, G., \& Willems, R. M. (2009). Semantic unification. In Gazzaniga, M. (Ed.), The Cognitive Neurosciences, 4th ed. (pp. 819-836). Cambridge, MA: MIT Press.

Hamilton, L. S., Edwards, E., \& Chang, E. F. (2018). A spatial map of onset and sustained responses to speech in the human superior temporal gyrus. Current Biology, 28(12), 18601871.

Hasson, U., Egidi, G., Marelli, M., \& Willems, R. M. (2018). Grounding the neurobiology of language in first principles: The necessity of non-language-centric explanations for language comprehension. Cognition, 180, 135-157.

Hasson, U., \& Nusbaum, H. C. (2019). Emerging opportunities for advancing cognitive neuroscience. Trends in Cognitive Sciences, 23(5), 363-365.

Heim, I., \& Kratzer, A. (1998). Semantics in Generative Grammar. Oxford: Blackwell.

Herfeld, C., \& Lisciandra, C. (2019). Knowledge transfer and its contexts. Studies in History and Philosophy of Science Part A, 77, 1-10.

Hickok, G., \& Poeppel, D. (2016). Neural basis of speech perception. In Hickok, G., \& Small, S. (Eds.), Neurobiology of Language. London: Academic Press, 299-310.

Hullett, P. W., Hamilton, L. S., Mesgarani, N., Schreiner, C. E., \& Chang, E. F. (2016). Human superior temporal gyrus organization of spectrotemporal modulation tuning derived from speech stimuli. Journal of Neuroscience, 36(6), 2014-2026.

Jackendoff, R. (1983). Semantics and Cognition. Cambridge, MA: MIT Press.

Jackendoff, R. (2002). Foundations of Language: Brain, Meaning, Grammar, Evolution. Oxford: Oxford University Press.

Jackendoff, R. (2007). A parallel architecture perspective on language processing. Brain Research, 1146, 2-22.

Jakobson, R., \& Halle, M. (1971). Fundamentals of Language. The Hague: Mouton.

Jasmin, K., Lima, C. F., \& Scott, S. K. (2019). Understanding rostral-caudal auditory cortex contributions to auditory perception. Nature Reviews Neuroscience, 20(7), 425-434. 
Katz, J. J., \& Fodor, J. A. (1963). The structure of a semantic theory. Language, 39(2), 170210.

Kim, A., \& Osterhout, L. (2005). The independence of combinatory semantic processing: Evidence from event-related potentials. Journal of Memory and Language, 52(2), 205-225.

Kuperberg, G. R., Choi, A., Cohn, N., Paczynski, M., \& Jackendoff, R. (2010). Electrophysiological correlates of complement coercion. Journal of Cognitive Neuroscience, 22(12), 2685-2701.

Levelt, W. J. (2012). A History of Psycholinguistics: The Pre-Chomskyan Era. Oxford: Oxford University Press.

Lyu, B., Choi, H. S., Marslen-Wilson, W. D., Clarke, A., Randall, B., \& Tyler, L. K. (2019). Neural dynamics of semantic composition. Proceedings of the National Academy of Sciences, 116(42), 21318-21327.

Marantz, A. (2005). Generative linguistics within the cognitive neuroscience of language. The Linguistic Review, 22(2-4), 429-445.

Marr, D. (1982). Vision: A Computational Investigation into the Human Representation and Processing of Visual Information. San Francisco: Freeman.

Marr, D., \& Poggio, T. (1976). From understanding computation to understanding neural circuitry. MIT AI Memos 357: 1-22.

Martin, A. E., \& Doumas, L. A. (2019). Predicate learning in neural systems: using oscillations to discover latent structure. Current Opinion in Behavioral Sciences, 29, 77-83.

Martin, A. E., \& Baggio, G. (2019). Modelling meaning composition from formalism to mechanism. Philosophical Transactions of the Royal Society B: Biological Sciences 375 (1791), 1-7.

Mesgarani, N., Cheung, C., Johnson, K., \& Chang, E. F. (2014). Phonetic feature encoding in human superior temporal gyrus. Science, 343(6174), 1006-1010.

Michalon, 0., \& Baggio, G. (2019). Meaning-driven syntactic predictions in a parallel processing architecture: Theory and algorithmic modeling of ERP effects. Neuropsychologia, 131, 171-183.

Moro, A. (2016). Impossible Languages. Cambridge, MA: MIT Press.

Moro, A. (2019). The geometry of predication: A configurational derivation of the defining property of clause structure. Philosophical Transactions of the Royal Society B: Biological Sciences 375(1791).

Musso, M., Moro, A., Glauche, V., Rijntjes, M., Reichenbach, J., Büchel, C., \& Weiller, C. (2003). Broca's area and the language instinct. Nature Neuroscience, 6(7), 774. 
Nelson, M. J., El Karoui, I., Giber, K., Yang, X., Cohen, L., Koopman, H., Cash, S.S., Naccache, L., Hale, J.T., Pallier, C., \& Dehaene, S. (2017). Neurophysiological dynamics of phrasestructure building during sentence processing. Proceedings of the National Academy of Sciences, 114(18), E3669-E3678.

Nieuwland, M.S., Barr, D.J., Bartolozzi, F., Busch-Moreno, S., Darley, E., Donaldson, D.I., Ferguson, H.J., Fu, X., Heyselaar, E., Huettig, F., Husband, E.M., Ito, A., Kazanina, N., Kogan, V., Kohút, Z., Kulakova, E., Mézière, D., Politzer-Ahles, S., Rousselet, G., Rueschemeyer, S.-A., Segaert, K., Tuomainen, J., \& von Grebmer Zu Wolfsthurn, S. (2019). Dissociable effects of prediction and integration during language comprehension: Evidence from a large-scale study using brain potentials. Philosophical Transactions of the Royal Society B, 375(1791).

Nowak, I., \& Baggio, G. (2017). Developmental constraints on learning artificial grammars with fixed, flexible and free word order. Frontiers in Psychology, 8, 1816.

Olstad, A. M. H., Fritz, I., and Baggio, G. (2020). Composition decomposed: Distinct neural mechanisms support processing of nouns in modification and predication contexts. Journal of Experimental Psychology: Learning, Memory, and Cognition 46 (11): 2193-2206.

Paczynski, M., Jackendoff, R., \& Kuperberg, G. (2014). When events change their nature: The neurocognitive mechanisms underlying aspectual coercion. Journal of Cognitive Neuroscience, 26(9), 1905-1917.

Papeo, L., Lingnau, A., Agosta, S., Pascual-Leone, A., Battelli, L., \& Caramazza, A. (2014). The origin of word-related motor activity. Cerebral Cortex, 25(6), 1668-1675.

Partee, B. H. (2018). Changing notions of linguistic competence in the history of formal semantics. In Ball, D. \& Rabern, B. (Eds.), The Science of Meaning: Essays on the Metatheory of Natural Language Semantics, 172-196. Oxford University Press.

Patterson, K., Nestor, P. J., \& Rogers, T. T. (2007). Where do you know what you know? The representation of semantic knowledge in the human brain. Nature Reviews Neuroscience, 8(12), 976.

Pavan, A., \& Baggio, G. (2013). Linguistic representations of motion do not depend on the visual motion system. Psychological Science, 24(2), 181-188.

Petersson, K. M., \& Hagoort, P. (2012). The neurobiology of syntax: Beyond string sets. Philosophical Transactions of the Royal Society B: Biological Sciences, 367(1598), 19711983.

Poeppel, D., \& Embick, D. (2005). Defining the relation between linguistics and neuroscience. In Cutler, A. (Ed.), Twenty-first Century Psycholinguistics. Mahwah, NJ: Lawrence Erlbaum Associates, 103-118.

Pulvermüller, F. (2010). Brain embodiment of syntax and grammar: Discrete combinatorial mechanisms spelt out in neuronal circuits. Brain and Language, 112(3), 167-179. 
Pulvermüller, F., Cappelle, B., \& Shtyrov, Y. (2013). Brain basis of meaning, words, constructions, and grammar. In Hoffmann, T. \& Trousdale, G. (Eds.) The Oxford Handbook of Construction Grammar. Oxford: Oxford University Press, 397-416.

Pustejovsky, J. (1995). The Generative Lexicon. Cambridge, MA: MIT Press.

Rabovsky, M., Hansen, S. S., \& McClelland, J. L. (2018). Modelling the N400 brain potential as change in a probabilistic representation of meaning. Nature Human Behaviour, 2(9), 693.

Rapp, A. M., Mutschler, D. E., \& Erb, M. (2012). Where in the brain is nonliteral language? A coordinate-based meta-analysis of functional magnetic resonance imaging studies. Neuroimage, 63(1), 600-610.

Ristad, E. S. (1993). The Language Complexity Game. Cambridge, MA: MIT Press.

Sejnowski, T. J., Koch, C., \& Churchland, P. S. (1988). Computational neuroscience. Science, 241(4871), 1299-1306.

Shieber, S. M. (1986/2003). An Introduction to Unification-based Approaches to Grammar. Stanford, CA: CSLI Publications.

Snijders, T. M., Vosse, T., Kempen, G., Van Berkum, J. J., Petersson, K. M., \& Hagoort, P. (2008). Retrieval and unification of syntactic structure in sentence comprehension: an fMRI study using word-category ambiguity. Cerebral Cortex, 19(7), 1493-1503.

Steedman, M. (1992). Grammars and Processors. Technical Report MS-CIS-92-52. Department of Computer and Information Science, University of Pennsylvania.

Tang, C., Hamilton, L. S., \& Chang, E. F. (2017). Intonational speech prosody encoding in the human auditory cortex. Science, 357(6353), 797-801.

Townsend, D. J., Bever, T. G., \& Bever, T. G. (2001). Sentence Comprehension: The Integration of Habits and Rules. Cambridge, MA: MIT Press.

Trotzke, A., \& Zwart, J. W. (2014). The complexity of narrow syntax: Minimalism, representational economy, and simplest Merge. In Newmeyer, F. J., \& Preston, L. B. (Eds.). (2014). Measuring Grammatical Complexity. Oxford: Oxford University Press, 128-147.

Trubetzkoy, N. S. (1969). Principles of Phonology. Berkeley, CA: University of California Press.

Urbach, T. P., DeLong, K. A., \& Kutas, M. (2015). Quantifiers are incrementally interpreted in context, more than less. Journal of Memory and Language, 83, 79-96.

Vulchanova, M., Milburn, E., Vulchanov, V., \& Baggio, G. (2019). Boon or Burden? The role of compositional meaning in figurative language processing and acquisition. Journal of Logic, Language and Information, 28(2), 359-387.

Yang, C. D. (2002). Knowledge and Learning in Natural Language. Oxford: Oxford University Press. 
974 Yang, C. D. (2004). Universal Grammar, statistics or both? Trends in Cognitive Sciences, 975 8(10), 451-456.

976 Zaccarella, E., Meyer, L., Makuuchi, M., \& Friederici, A. D. (2017). Building by syntax: the 977 neural basis of minimal linguistic structures. Cerebral Cortex, 27(1), 411-421.

978 Van Dongen, A. M. (1996). A new algorithm for idealizing single ion channel data containing 979 multiple unknown conductance levels. Biophysical Journal, 70(3), 1303-1315.

980 Van Rooij, I. (2008). The tractable cognition thesis. Cognitive Science, 32(6), 939-984.

981 Van Rooij, I., \& Baggio, G. (2020). Theory before the test: How to build high-verisimilitude 982 explanatory theories in psychological science. PsyArXiv preprint, 10.31234/osf.io/7qbpr.

983 Westerlund, M., \& Pylkkänen, L. (2017). How Does the Left Anterior Temporal Lobe 984 Contribute to Conceptual Combination? Interdisciplinary Perspectives. In J. A. Hampton \& 985 Y. Winter (Eds.), Compositionality and Concepts in Linguistics and Psychology. New York, 986 NY: Springer, 269-290.

987 Wittenberg, E., Paczynski, M., Wiese, H., Jackendoff, R., \& Kuperberg, G. (2014). The 988 difference between 'giving a rose' and 'giving a kiss': Sustained neural activity to the light 989 verb construction. Journal of Memory and Language, 73, 31-42. 